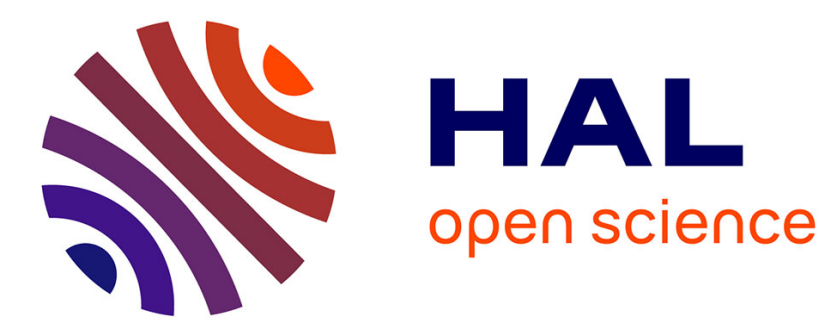

\title{
Pascal et la maladie de la pensée Jean-Christophe Cavallin
}

\section{- To cite this version:}

Jean-Christophe Cavallin. Pascal et la maladie de la pensée. Libres cahiers pour la psychanalyse, 2013, 28 (2), 10.3917/lcpp.028.0143 . hal-01766949

\section{HAL Id: hal-01766949 \\ https://hal.science/hal-01766949}

Submitted on 14 Apr 2018

HAL is a multi-disciplinary open access archive for the deposit and dissemination of scientific research documents, whether they are published or not. The documents may come from teaching and research institutions in France or abroad, or from public or private research centers.
L'archive ouverte pluridisciplinaire HAL, est destinée au dépôt et à la diffusion de documents scientifiques de niveau recherche, publiés ou non, émanant des établissements d'enseignement et de recherche français ou étrangers, des laboratoires publics ou privés. 


\section{PASCAL ET LA MALADIE DE LA PENSÉE \\ Jean-Christophe Cavallin}

In Press | « Libres cahiers pour la psychanalyse »

2013/2 N² 28 | pages 143 à 160

ISSN $1625-7480$

ISBN 9782848352671

Article disponible en ligne à l'adresse :

https://www.cairn.info/revue-libres-cahiers-pour-lapsychanalyse-2013-2-page-143.htm

\section{Pour citer cet article :}

Jean-Christophe Cavallin, «Pascal et la maladie de la pensée », Libres cahiers pour la psychanalyse 2013/2 ( $\left.\mathrm{N}^{\circ} 28\right)$, p. 143-160.

DOI 10.3917/lcpp.028.0143

Distribution électronique Cairn.info pour In Press.

(C) In Press. Tous droits réservés pour tous pays.

La reproduction ou représentation de cet article, notamment par photocopie, n'est autorisée que dans les limites des conditions générales d'utilisation du site ou, le cas échéant, des conditions générales de la licence souscrite par votre établissement. Toute autre reproduction ou représentation, en tout ou partie, sous quelque forme et de quelque manière que ce soit, est interdite sauf accord préalable et écrit de l'éditeur, en dehors des cas prévus par la législation en vigueur en France. Il est précisé que son stockage dans une base de données est également interdit. 


\title{
Pascal et la maladie de la pensée
}

\author{
Jean-Christophe Cavallin
}

I shall be like that tree; I shall die from the top.

Jonathan Swift

For I had gravidum cor, foetum caput, a kind of imposthume in my head, which I was very desirous to be unladen of.

Robert Burton, The Anatomy of Melancholy

N MAI 1790, LORS D’UNE PROMENADE AU LIDO, Goethe trouve un
crâne de bélier dans le sable du cimetière israélite. Freud évoque cet épisode dans une lettre à Fliess du 4 octobre 1897. Devant le vestige blanchi par le temps que son domestique lui présente, le poète naturaliste a l'intuition de sa théorie du crâne vertébral : la boîte crânienne comme épanouissement de la dernière vertèbre sous la poussée de la moelle épinière. Ce processus d'accroissement (Steigerung) ou d'hypertrophie médullaire à l'origine du cerveau des vertébrés supérieurs confirme son hypothèse d'une morphogénèse continue déduisant toutes les espèces animales d'un Urtier ${ }^{1}$ originel. L'enthousiasme scientifique de Goethe, confiant dans la plasticité et dans le perfectionnement des organismes naturels, offre le pendant optimiste de la pensée de Rousseau

1. Archi-animal. 
selon laquelle «L'homme qui pense est un animal malade ». Dans l'optique du penseur de Genève, il conviendrait de parler, plutôt que d'épanouissement, d'une poussée inflammatoire de l'organe de la sensibilité. L'homme serait un animal à la cervelle enflammée, dont les pénibles cogitations ruinent le fragile bonheur. Dans son traité De l'hypocondrie et de la mélancolie, sur les traces de L'homme de génie et la mélancolie du pseudo-Aristote, Duvivier définit l'hypocondrie, naturelle aux hommes de Lettres, comme un mal résultant d'une «surexcitation cérébrale ${ }^{2} \gg$ ou « irritation du cerveau » changeant l'organe de la pensée en un «centre phlogosé $e^{3}$ » qui absorbe les forces du «principe sentant » et finit par « produire de graves altérations ${ }^{4}$ » dans tout l'organisme. Duvivier suggère l'existence d'une forme d'hypocondrie dont « [le] siège soit exclusivement dans le cerveau ${ }^{5} »$. Nous voudrions esquisser une étiologie de ce morbus capitis ou « maladie de la tête » en ouvrant un dialogue entre, d'un côté, les hypothèses de Freud sur l'hypocondrie et, de l'autre, la théorie de la «pensée » dont Pascal esquisse le drame - en forme d'objection à la res cogitans de Descartes - dans les deux figures allégoriques du «membre pensant» et du « roseau pensant ».

Un trait singulier de Freud - un trait propre à son génie et à toute science nouvelle (toute description d'objets nouveaux) - est son art de l'analogie. Dans Pour introduire le narcissisme, il établit un parallèle entre l'organe affecté d'une maladie non organique et le «modèle » bien connu «d'un organe douloureusement sensible, modifié en quelque façon sans être pourtant malade au sens habituel : [...] l'organe génital en état d'excitation ${ }^{6} »$. La douleur hypocondriaque résulterait d'une érogénéisation - ou surinvestissement libidinal - de l'organe souffrant, hypertrophié et psychisé par la libido du moi. Laissant de côté la belle

2. E. Duvivier, De l'hypocondrie et de la mélancolie, Paris, Chez l'Auteur, Rue Louis-leGrand, 1853, p. 15.

3. Ibid., p. 26.

4. Ibid., p. 15 .

5. Ibid., p. 14.

6. S. Freud, Pour introduire le narcissisme, Payot, « Petite Bibliothèque Payot », 2012, p. 54 . 
perspective qu'ouvre cette analogie sur la possibilité de retourner la comparaison freudienne (1'hypocondrie comme génitalisation de l'organe malade) et de penser en retour la pulsion sexuelle comme hypocondrie génitale, nous nous intéresserons à la valeur de «modèle » que Freud attribue à l'organe génital, "constitué en prototype de tout organe $^{7} »$. Le même postulat se retrouve dans l'analyse de l'érogénéité proposée par Pulsions et destins des pulsions (« toute zone du corps peut se comporter comme des organes génitaux secondaires $\left.{ }^{8} »\right)$ et Freud compte au nombre de ces parties du corps, susceptibles d'être érotisées, l'activité cérébrale elle-même, à l'envi « travail intellectuel » ou « curiosité intellectuelle » conçus comme sexualisation de la pensée ou sublimation de la libido du moi. Le cerveau génitalisé de Freud et le cerveau phlogosé de Duvivier jouissent ou souffrent tous les deux d'une même irritabilité due à la surexcitation de leur fonctionnement normal : leur turgescence hyperactive présuppose un détournement ou une captation forcée des forces vives de la psyché.

Il y aurait donc un lien entre l'organe génital, l'hypocondrie et la pensée. Les fragments de Pascal sur les «membres pensants » articulent dialectiquement cette triade de motifs :

368. Membres. Commencer par là. Pour régler l'amour qu'on se doit à soimême il faut s'imaginer un corps plein de membres pensants, car nous sommes membres du tout, et voir comment chaque membre devrait s'aimer, etc.

371. Qu'on s'imagine un corps plein de membres pensants.

372. Être membre est n'avoir de vie, d'être et de mouvement que par l'esprit du corps. Et pour le corps, le membre séparé ne voyant plus le corps auquel il appartient n'a plus qu'un être périssant et mourant. Cependant il croit être un tout et ne se voyant point de corps dont il dépende, il croit ne dépendre que de soi et veut se faire centre et corps lui-même. Mais n'ayant point en soi de principe de vie il ne fait que s'égarer et s'étonne dans

7. P. Fédida, «L'Hypocondrie de l'expérience du corps», in Traité de psychologie de l'adulte. Psychopathologie des limites, dir. Catherine Chabert, Dunod, 2009, p. 94.

8. S. Freud, Pulsions et destins des pulsions, Payot, « Petite Bibliothèque Payot », 2012, p. 35 . 
l'incertitude de son être, sentant bien qu'il n'est pas corps, et cependant ne voyant point qu'il soit membre d'un corps. Enfin quand il vient à se connaître il est comme revenu chez soi et ne s'aime plus que pour le corps. Il plaint ses égarements passés. [...]

373. Il faut n'aimer que Dieu et ne haïr que soi. Si le pied avait toujours ignoré qu'il appartînt au corps et qu'il y eût un corps dont il dépendît, s'il n'avait eu que la connaissance et l'amour de soi et qu'il vînt à connaître qu'il appartient à un corps duquel il dépend, quel regret, quelle confusion de sa vie passée, d'avoir été inutile au corps qui lui a influé la vie, qui l'eût anéanti s'il l'eût rejeté et séparé de soi, comme il se séparait de lui ${ }^{9}$.

La parabole pascalienne représente le corps christique comme un corps désintégré, agité de «membres pensants ». La pathologie de ce corps figure le cauchemar d'une hypocondrie généralisée où une espèce de pensée - quelque chose de " pensant »-s'enkysterait dans chaque organe, éternel souci d'un faux soi obsédé par sa propre mort et désorganisant le tout. La maladie de ce corps dont le pied ou la main sont des « membres pensants » est avant tout la maladie du pied ou de la main qui pensent : le membre étant privé de toute substance («n'ayant point en soi de principe de vie »), mais ne pouvant se penser sans se penser comme un tout («il croit être un tout»), la pensée du «membre pensant» est une pensée désemparée par l'angoisse de la mort : il s'étonne et s'épouvante d'être «périssant et mourant » parce qu'il se pense séparé et sevré de l'organisme dont il méconnaît l'existence. Le désespoir égaré de cette pensée prodigue, éloignée du corps qui lui donne vie, est la réponse de Pascal aux Méditations de Descartes et à sa «res cogitans». Pour Descartes, la pensée est non pas une qualité, mais la substance du moi. Elle se suffit à elle-même et suffit à démontrer de façon indubitable l'existence de l'ego. C'est la signification du «cogito, sum»: la pensée soutient l'existence - et comme substance et comme argument. Or, dans les Pensées de Pascal, 1'homme n'est pas une « res », mais un «membrum» (cogitans) : sa pensée est un attribut, une qualité adjective, mais sa substance est d'être membre et donc, automatiquement - en tant qu'il n'est pas un tout -, de ne pas avoir de substance. En tant qu'il se pense comme

9. Pascal, Pensées, éd. Lafuma. 
" centre et corps de lui-même », sa pensée crée en lui l'illusion d'un « faux self», illusoirement autonome, mais endeuillé du tout dont il nie l'existence et ressassant sans repos le pressentiment de sa mort.

La parabole pascalienne donne corps, littéralement, à la synecdoque hypocondriaque : la vie psychique du corps divin y est tout entière engloutie, appauvrie et desséchée dans l'hypertrophie du membre pensant. La pensée souffrante de l'organe - totalité imaginaire à la fois sevrée et sevrante - est le vestige somatisé d'une totalité perdue. Elle enclave dans le corps christique l'abcès tumide d'un faux tout, dont la forclusion psychique fonctionne comme déni d'une désintégration. La synecdoque hypocondriaque est une figure atrophiée dans laquelle s'est enrayé le principe du pars pro toto. Séparée de l'esprit qui la vivifierait, sa figuralité demeure lettre morte : au contraire de signifier la totalité vivante du corps, la mort-vivance de l'organe en signe l'effondrement.

Pensée de «l'homme sans Dieu », la pensée du «membre pensant» entretient avec l'Esprit (ou «pensée du corps » christique) le même rapport que l'amour-propre entretient avec la Charité (ou «amour en Dieu ») :

Si le pied avait toujours ignoré qu'il appartînt au corps et qu'il y eût un corps dont il dépendît, s'il n'avait eu que la connaissance et l'amour de soi et qu'il vînt à connaître qu'il appartient à un corps duquel il dépend, quel regret, quelle confusion de sa vie passée, d'avoir été inutile au corps qui lui a influé la vie [...].

La pensée et l'amour-propre ( «la connaissance et l'amour de soi »), en tant qu'ils surinvestissent ou hypertrophient un restant (le «moi » comme reste et vestige d'une « première nature » ou d'une totalité), sont des formations de défense : leur suractivité morbide s'épuise à démentir le cataclysme psychique d'un désinvestissement total lié à l'effondrement ou à la perte du tout. Leur logique opératoire n'est pas une logique du pars pro toto: la partie pour le tout (synecdoque intégrative); mais bien une logique du pars pro nihilo : la partie plutôt que rien (synecdoque désintégrée). C'est la raison pour laquelle, dans le texte de Pascal, 
la pensée prend toujours la forme d'une affection mélancolique. Il faut relire le magnifique fragment sur le «Divertissement» (Laf. 136) : dès qu'il n'est plus diverti, l'homme se met à penser; et dès qu'il se met à penser, sa pensée ne sécrète en lui que doutes, angoisses, frayeurs, fantômes de maladie et cogitations morbides. Même le plus heureux des hommes, s'il a le loisir d'y penser, devient le plus malheureux :

Le roi est environné de gens qui ne pensent qu'à divertir le roi et à l'empêcher de penser à lui. Car il est malheureux, tout roi qu'il est, s'il y pense. [...] Qu'on en fasse les preuves, qu'on laisse un roi tout seul sans aucune satisfaction des sens, sans aucun soin dans l'esprit, sans compagnie et sans divertissements, penser a lui tout à loisir, et l'on verra qu'un roi sans divertissement est un homme plein de misères.

Le tableau clinique que Pascal donne de la pensée normale, laquelle ne produit d'elle-même et sans nulle cause de chagrin que des spectres de désastres, de mort ou de maladie, est fortement inspiré du tableau que donne Robert Burton de la pensée mélancolique : "desease of an inflamed brain ${ }^{10}$ " dont les symptômes sont de "continual and superfluous cogitations, [...] immoderate perturbations of the mind, fear, sorrow, \&c. ${ }^{11} \gg$. Et le remède prescrit est la vieille panacée figurant dans tous les traités sur l'humeur mélancolique : le « divertissement» sous toutes ses formes - jeu, chasse, danse, musique, déplacements, emplois publics.

La pensée selon Pascal a donc tous les attributs d'une « mélancolie de la tête » (Head-melancholy), c'est-à-dire d'une hypocondrie sans autre support organique que le siège de la pensée. La leçon freudienne supposerait que l'on trouve à l'origine de sa surexcitation morbide un processus d'érogénéisation pathogène. Or, le modèle génital (notre troisième motif) configure in absentia l'ensemble des fragments sur le «membre pensant » (Laf. 368-371). S'il reste implicite - pour des raisons de bienséance et de contexte apologétique - dans l'énoncé de la parabole pascalienne, il est massivement présent dans la source

10. Robert Burton, The Anatomy of Melancholy, I-II-IV-3.

11. Ibid., I-III-II-1. 
augustinienne du texte. Pascal semble avoir déduit la figure allégorique du « membre pensant» d'une hybridation de la «(res) cogitans » de Descartes et du «membrum (genitale) » dont saint Augustin écrit 1'histoire dans le quatorzième livre de La Cité de Dieu.

Avant le péché, Adam et Ève ne connaissaient pas leur nudité parce que leur volonté ne faisait qu'une avec leur corps. Leurs organes génitaux répondaient comme les autres à leur volonté efficiente : « la concupiscence ne sollicitait pas encore ces membres malgré la volonté (nondum libido membra illa praeter arbitrium commovebat) ${ }^{12} \gg$. Le péché détraque tout : à peine l'homme a-t-il désobéi à Dieu, à peine sa volonté a-t-elle fait sécession, que son corps lui désobéit et qu'il sent dans sa chair des mouvements étranges, absolument hors de contrôle et aliénant son corps propre : «Ils sentirent un mouvement inconnu dans leur chair désobéissante (senserunt ergo novum motum inoboedientis carnis suae $)^{13}$ »; " Cette désobéissance qui soustrait les membres génitaux (genitalia corporis membra) à la puissance de la volonté, ne publiet-elle pas le salaire de la désobéissance de 1'homme ${ }^{14}$ ? » Si l'homme peut encore mouvoir sa main ou son pied au gré de sa volonté, c'est la concupiscence («libido »), un agent inconnu, qui meut ses organes génitaux ; et l'homme voile sa nudité pour dissimuler sa honte ${ }^{15}$. Avant le péché, Adam en eût-il exprimé la volonté, ce fidèle serviteur ${ }^{16}$ qu'était son « membre génital» aurait rempli son ministère et « ensemencé le champ naturel comme la main ensemence le champ (ita genitale arvuum

12. Saint Augustin, La Cité de Dieu, éd. Jean-Claude Eslin, Paris, Seuil, « Points Sagesses », vol. 2, XIV, XVII, p. 176. Le traducteur du texte emploie toutes sortes de périphrases pour désigner ce que saint Augustin appelle systématiquement les « membra genitalia ». Dans la suite de notre étude, nous nous permettrons d'éliminer tout aussi systématiquement les circonlocutions du traducteur et de revenir à la leçon augustienne en traduisant littéralement « membra genitalia » par « membres génitaux ».

13. Ibid., XIV, XIII.

14. Ibid., XIV, XX, p. 181.

15. «Confus de la rébellion de leur chair, témoin pénal de leur propre rébellion (confusi inoboedientia carnis suae, tamquam teste poena inoboedientiae suae), ils entrelaçent des feuilles de figuier dont il se font comme des ceintures » (XIV, XVII, p. 177).

16. « Doit-il nous sembler étrange que, dans l'état d'innocence, ce membre obéisse sans nulle concupiscence (sine ista libidine) à la volonté qui commande impérieusement à tant d'autres organes? Ne remuons-nous pas quand nous voulons la main ou le pied pour 
vas in hoc opus creatum seminaret, ut nunc terram manus ${ }^{17}$ ) ». Après le péché, le même membre génital n'obéit plus qu'aux mouvements de la concupiscence («libidinis motibus », «libidinis appetitus »), qui le redresse ou le rendort malgré la volonté d'Adam (« non omni modo ad arbitrium nostrum movet aut non movet $\left.^{18} »\right)$.

De l'intégrité perdue du corps adamique à la désintégration du corps christique, le « membre génital » soumis aux « mouvements de la concupiscence (motibus libidinis) » a donc servi d'archétype à l'allégorie du « membre pensant», corollaire polémique de la «res cogitans» des Méditations. Si le pied pensant de Pascal, figure de l'homme qui pense et se pense comme ego, est une transposition du «membre génital» augustinien, il en résulte automatiquement que la « pensée » agissante en l'homme et le sevrant du corps divin, en tant qu'elle pose une équation entre pensée et existence (cogito, sum), est une forme dérivée de la même « libido » qui régit les mouvements du membre de la génération. C'est parce qu'elle est de nature libidinale que la pensée, chez Pascal, sèvre le « membre pensant » (i.e. l'homme) de l'esprit qui irrigue et fait vivre le corps. Enkystement cérébral de la libido, érogénéisation hypocondriaque de l'âme adamique, la pensée « se comporte comme un organe génital secondaire » (le membrum genitale d'Augustin) et provoque en l'homme un état de schisme et de désintégration. Cette surexcitation morbide des facultés intellectuelles a un nom dans la psychologie augustinienne comme dans le texte pascalien : c'est la libido sciendi, un des trois avatars de la concupiscence (libido sentiendi, libido dominandi, libido sciendi ${ }^{19}$ ). L'homme qui pense trop (mais, chez Pascal, tout

accomplir l'acte qui réclame leur ministère ? » (XIV, XXIII, p. 183) Autrement dit, sans le péché, «l'homme eût semé, la femme eût recueilli, quand il eût fallu, autant qu'il eût fallu : les membres génitaux (genitalibus membris) eussent obéi au mouvement de la volonté et non à l'aiguillon de la concupiscence (voluntate motis, non libidine concitatis)» (XIV, XXIV, p. 185).

17. Ibid., XIV, XX.

18. Ibid., XVII.

19. Cf. Laf. 545. « Tout ce qui est au monde est concupiscence de la chair ou concupiscence des yeux ou orgueil de la vie. Libido sentiendi, libido sciendi, libido dominandi. Malheureuse la terre de malédiction que ces trois fleuves de feu embrasent plutôt qu'ils n'arrosent. » 
homme qui pense est un homme qui pense trop), au même titre que l'homme charnel ou que l'homme intempérant, est donc un homme affecté d'un «mal de concupiscence » (libidinis morbus).

Pour comprendre l'étiologie de ce mal de la pensée, il faut être attentif à la logique du récit que saint Augustin donne de l'épisode catastrophique du péché originel. La première conséquence de la désobéissance d'Adam est qu'en se séparant de Dieu, il entre dans la mort. Son péché le désintègre : expulsé du tout dont il était une partie intégrante, il cesse automatiquement d'être le tout de ses parties. Cet état de déréliction dans lequel se retrouve l'homme comme partie désintégrée d'une totalité manquante, Augustin l'appelle : être « dans la mort» (« semper in morte $\left.{ }^{20} »\right)$, c'est-à-dire « jamais vivant, jamais mourant, mais mourant sans fin» («numquam viventes, numquam mortui, sed sine fine morientes ») - définition que Pascal résume dans le « périssant et mourant » qu'il applique au « membre pensant », c'est-à-dire à l'homme qui pense.

Adam entre dans la mort - livre XIII - et, aussitôt - livre XIV -, entre en scène la concupiscence. Dans la consécution des livres, la révolte du membre génital est la réponse du corps au cataclysme psychique de sa désintégration. La libido apparaît comme formation de défense. Son opération de saisie sur le membre génital investit réactivement une partie de l'organisme. L'érection incontrôlable, qui suit la désobéissance, totémise (et propitie) la cadavérisation de la nature adamique dans le priapisme d'un membre changé en organe total ou en pseudo-organisme régi par la libido.

L'image pascalienne du «roseau pensant» reprend le même processus de formation défensive, mais en déplace la topique : la même logique de totémisation défensive y fonctionne comme théorisation, non plus de l'irruption de la «libido » et de son enkystement dans le membre génital, mais de l'irruption de la «pensée » et de son enclavement dans le corps mourant de l'homme.

20. Ibid., XIII-XII. 
200. L'homme n'est qu'un roseau, le plus faible de la nature, mais c'est un roseau pensant. Il ne faut pas que l'univers entier s'arme pour l'écraser ; une vapeur, une goutte d'eau suffit pour le tuer. Mais quand l'univers l'écraserait, l'homme serait encore plus noble que ce qui le tue, puisqu'il sait qu'il meurt et l'avantage que l'univers a sur lui. L'univers n'en sait rien. $[\ldots]$

201. Le silence éternel de ces espaces infinis m'effraie ${ }^{21}$.

La définition pascalienne s'articule en trois moments : d'abord, «l'homme n'est qu'un roseau » - une parcelle de physis dans l'univers physique ; puis «le plus faible de la nature »-moment de son abolition entre les deux infinis, de sa disqualification dans l'échelle physique des forces ; et puis, «c'est un roseau pensant »-moment de sa conversion ou promotion qualitative du monde matériel ou physique (ordre de la chair) à l'univers intellectuel (ordre de l'esprit). Le scénario du fragment développe une idée simple : c'est quand l'univers l'écrase que le roseau agonisant s'échappe dans la pensée, faisant ainsi de sa mort et de la conscience de sa mort non pas la preuve de son néant, mais l'argument de son triomphe. Le drame est intellectuel : le roseau (c'est-à-dire l'homme) regarde l'espace infini qui l'engloutit dans son silence, dans la démesure effrayante des deux abîmes vertigineux de l'infiniment petit et de l'infiniment grand. Ce n'est plus le «monde clos » dont il croyait être le centre, mais un « univers infini » qui, ne formant plus un tout, n'a plus ni centre ni partie. Ce spectacle l'épouvante, écrase sa petitesse et lui fait subir l'agonie d'un irrémédiable abandon. À l'instant même où il s'abîme et se noie dans l'étendue qui l'anéantit comme un point (selon la loi mathématique, souvent rappelée par Pascal, qu'un point s'annule dans l'infini), le roseau se ressaisit dans la conscience de cette mort. Il enclave sa mort physique dans l'organe de la pensée, totalité intensive et d'un ordre supérieur qui comprend l'univers physique et englobe son extension :

113. Roseau pensant. Ce n'est point de l'espace que je dois chercher ma dignité, mais c'est du règlement de ma pensée. [...] Par l'espace l'univers me comprend et m'engloutit comme un point : par la pensée je le comprends.

21. Pascal, Pensées, éd. Lafuma. 
Même traumatisme, même réaction : l'univers engloutit l'homme qui, au moment de mourir, retire et enkyste son moi dans le siège de la pensée. L'expérience originaire est celle d'une absence de secours : l'homme se retrouve seul, abandonné dans l'univers, sans que rien ne lui réponde dans « le silence éternel de ces espaces infinis ». Cette déréliction dans 1'espace infini - «Qu'est-ce qu'un homme, dans l'infini ${ }^{22}$ ? »- est le thème central du long fragment sur la « Disproportion de 1 'homme ${ }^{23} »$ :

Car enfin qu'est-ce que l'homme dans la nature ? Un néant à l'égard de l'infini, un tout à l'égard du néant, un milieu entre rien et tout, infiniment éloigné de comprendre les extrêmes ; la fin des choses et leur principe sont pour lui invinciblement cachés dans un secret impénétrable. Également incapable de voir le néant d'où il est tiré et l'infini où il est englouti.

La vie physique du roseau qu'est l'homme devant l'infini se désintègre sous la pression du silence qui l'engloutit et, au moment de mourir, s'enclave dans un organe de l'activité duquel dépend fantasmatiquement la conservation posthume de son intégrité perdue. Le jeu de mots de Pascal explicite la conversion : l'univers « comprend » le roseau et en engloutit l'unité ; mais la pensée du roseau «comprend» et conçoit (con-capere) l'univers. Le roseau, par la pensée, se change donc en un tout qui intègre l'univers comme une de ses parties. Une part du tout qu'est le corps (l'organe de la pensée) survit à la mort du corps et fonctionne comme un tout - comprenant le corps, l'univers et jusqu'à sa propre agonie - dans une rumination sans fin mêlant deuil et dénégation du traumatisme existentiel dont elle est la conséquence. Le « roseau pensant » de Pascal pense sans fin son agonie. Sa cogitation perpétuelle maintient en vie le traumatisme qu'exclut et inclut sa dénégation. La pensée fait vivre l'homme dans le perpétuel souci et l'attente d'une mort dont elle est la conséquence et qui a donc déjà eu lieu.

22. Laf. 199. « Disproportion de l'homme ».

23. Ibid. 
La dialectique configurant les fragments sur le « roseau pensant» rappelle de façon saisissante l'étiologie de l'hypocondrie : la pensée y joue le rôle de la plainte hypocondriaque, laquelle enkyste dans un organe la figure « cryptée ${ }^{24}$ d'un tout qui n'a pas pu être introjecté dans les forces vives du moi. Ce mal fantôme que nourrit un reflux libidinal provoqué par un abandon, ce « fragment du soi grandiose ${ }^{25}$ » enclavé dans un organe, y perpétuent un «faux self» que l'on ne saurait guérir sans réveiller la terreur de l'effondrement psychique dont il est tout à la fois le stigmate et le déni (déni non interprétable, parce que corporéisé) ${ }^{26}$.

Dans les Pensées de Pascal, le traumatisme à l'origine de l'enclavement douloureux dans l'organe de la pensée n'est autre que le retrait de Dieu privant l'homme de toute substance et le laissant sans secours, en état de déréliction, dans «le silence éternel [des] espaces infinis ». La pensée de «l'homme sans Dieu » repousse le spectre d'une « cadavérisation du psychique » en surexcitant de façon morbide l'activité du membrum cogitans, sorte de pénis incastrable ou de "faux self» inguérissable, perpétuant le deuil impossible de la mort-vivance du psychisme.

La dialectique des Pensées articule en profondeur les deux figures analogiques du « roseau pensant» et du «membre pensant ». Ces jumeaux allégoriques illustrent les deux conversions d'une anagogie spirituelle permettant à l'homme de quitter l'univers physique du Deus absconditus et d'être rapatrié dans le sein du Dieu vivant. Le « roseau pensant» figure le passage de «l'ordre de la chair » à «l'ordre de l'esprit » : le roseau meurt comme roseau (entité physique) et se (re)plie dans la pensée (entité intellectuelle) pour se sauver de cette mort ou en

24. «Il faudrait [...] se demander comment l'organe dont se plaint le malade prend nom de foie, d'estomac, de cœur ou de poumon, tels des ex-voto qui seraient les figures "cryptées" ainsi enclavées par la plainte répétitive afin de conserver intrapsychiquement le parent vivant » (Pierre Fédida, "L'Hypocondrie de l'expérience du corps » in Traité de psychologie de l'adulte. Psychopathologie des limites, éd. cit., p. 96).

25. Sur l'organe malade de l'hypocondriaque comme vestige corporéisé ou « fragment du soi grandiose », voir le beau livre d'Heinz Kohut, Le Soi - La psychanalyse des transferts narcissiques, Puf, « Le fil rouge », 2004.

26. Sur le « modèle de l'enkystement» dans l'hypocondrie, voir les analyses de Pierre Fédida sur le « faux self protecteur » (P. Fédida, op. cit., p. 109-113). 
sauver quelque chose figurant une totalité. Le « membre pensant » figure le passage de « l'ordre de l'esprit » à «l'ordre de la charité » : le membre renonce à la pensée ${ }^{27}$ ou à la pensée solipsiste dont l'orgueil et la suffisance imaginaient le faux tout d'une fausse autonomie (le cogito cartésien) et se trouve réintégré dans le corps surnaturel du Christ, sensible au cœur et non à la raison, qui l'intègre comme membre ${ }^{28}$.

On remarque, dans le deux cas, que la pensée est en l'homme une enclave pathogène qui, au sens propre, manque de tout : la pensée du « roseau » porte le deuil traumatique de son intégration à la vie naturelle; la pensée du « membre » porte le deuil inconscient de son intégration à la vie surnaturelle. Expulsée et séparée des deux adhésions possibles au corps charnel de la physis ou au corps spirituel du Christ («adhaerens deos unus spiritus est ${ }^{29}$ », dit Pascal), la pensée pense en déshérence, moribonde et désincarnée. Comme la plainte hypocondriaque, sa cogitatio morbide témoigne toujours d'un sevrage : tout en empêchant l'homme de vivre, la pensée lui tient lieu de vie ou, disons, occupe les lieux de la vie qu'il a perdue. Elle inclut comme revenance les deux formes de vie qu'elle exclut (vie naturelle dans le corps et vie surnaturelle en Dieu) : d'un côté, le roseau pensant revit sans fin dans sa pensée la mort du roseau physique ; de l'autre, l'homme pensant conserve dans sa pensée comme « la trace toute vide ${ }^{30} »$ et « le reste de la grandeur de $\left[\mathrm{sa}\right.$ ] première nature ${ }^{31} »$. Dans l'anthropologie pascalienne, bien plus que la vie physique, la véritable incarnation que l'homme enkyste dans sa pensée est la « vie en Dieu » d'Adam avant la

27. Laf. 374. « Si les pieds et les mains avaient une volonté particulière, jamais ils ne seraient dans leur ordre qu'en soumettant cette volonté particulière à la volonté première qui gouverne le corps entier. » Et encore, Laf. 372 : « Mais en aimant le corps il s'aime soi-même parce qu'il n'a d'être qu'en lui, par lui et pour lui. Qui adhaeret deo unus spiritus est. »

28. Sur les « trois ordres » dans les Pensées, voir en particulier Laf. 308. Pascal y compare les grandeurs infiniment différentes de la vie physique ou naturelle (la grandeur des rois et autres puissances de ce monde), de la vie intellectuelle (la grandeur des sages et savants) et de la vie spirituelle ou surnaturelle (la grandeur des saints) : "ordre de la chair », « ordre de l'esprit » et « ordre du cœur » (ou « ordre de la charité »).

29. Laf. 372. «Adhaerens deo unus spiritus est; on s'aime parce qu'on est membre de JésusChrist ; on aime Jésus-Christ parce qu'il est le corps dont on est membre. Tout est un. L'un est en l'autre comme les trois personnes. »

30. Laf. 148.

31. Laf. 136. 
désobéissance qui le vida de sa substance et lui apprit en même temps à penser et à mourir. Contrairement au cogito cartésien, la pensée selon Pascal est la preuve et le produit d'une absence de «principe de vie » et d'un défaut d'existence : cogito, ergo non sum. Elle prouve la mort de l'être psychique qui la hante comme un revenant et dont son éternel souci « panse » désespérément l'inguérissable survivance ${ }^{32}$.

À propos de la douleur psychisée de l'organe hypocondriaque, Ferenczi évoque l'activité d'un « tératome ${ }^{33}$ » intrapsychique consécutif à des traumatismes précoces. Une trop violente sensation d'abandon aurait entraîné cet enkystement de la psyché dans certaines parties du corps dont l'activité morbide aurait pour principal objet de proclamer cet abandon comme «nul et non advenu ${ }^{34} »$. Le terme de tératome, emprunté à l'embryologie, désigne une tumeur dont l'apparition rappelle un stade antérieur du développement de l'embryon. Dans l'organe hypocondriaque de Ferenzci survivrait donc un « jumeau » mort, un Soi «brutalement détruit », embaumé ou fossilisé ${ }^{35}$ dans une partie de l'organisme et dont la préservation, douloureuse et surinvestie, priverait le reste du moi des forces vives de la libido.

La préface de The Anatomy of Melancholy donne une formule saisissante de ce « tératome » dont l'activité morbide est à l'origine des sécrétions et effluves mélancoliques dont Burton se lamente parce qu'elles désespèrent sa vie :

For I had gravidum cor, foetum caput, a kind of imposthume in my head, which I was very desirous to be unladen of, and could imagine no fitter evacuation than this. («Car j'avais gravidum cor - [le cour lourd et la tête

32. C'est le « jeu de mots » dont parle Rimbaud dans la célèbre lettre à Izambard : «C'est faux de dire : Je pense : on devrait dire : On me pense. - Pardon du jeu de mots ["On me pense" = "On me panse"]. - Je est un autre. » (Lettre à Izambard, 13 mai 1871). Le « Je » qui pense dans ma pensée est un "Je » perdu qui me «panse », c'est-à-dire qui guérit mon deuil du fantasme de sa survie.

33. S. Ferenczi, Euvres complètes, Payot, 1977, tome 4, p. 96. Pour une étude de ce texte, voir G. Burloux, Le Corps et sa douleur, Dunod, 2004, chap. 5 : «Une douleur fossile : 1'hypocondrie », p. 79-103.

34. Ibid., p. 106.

35. Sur la « douleur fossile » de l'organe hypocondriaque, cf. G. Burloux, op. cit. (chap. 5). 
infectée, une sorte de tumeur dans la tête dont j'étais très désireux de me décharger, et je ne pouvais imaginer meilleure façon de le faire $\left.{ }^{36} . »\right)$

La métaphore de Burton - laquelle n'est, à tout prendre, pas beaucoup plus métaphorique que celle de « tératome » dont Ferenczi écrivait qu'il ne fallait pas y voir une «licence poétique ${ }^{37} »-$ localise la source de la maladie mélancolique dans « a kind of imposthume in the head». Le mal aurait donc un siège somatique et ce siège serait «foetum caput» : une tête infectée, un cerveau lésé par « une sorte de tumeur ». La plainte hypocondriaque de Burton produit ainsi l'illusion d'une affection organique. Son cerveau malade abrite un fragment tératophore produisant des pensées morbides qui tyrannisent sa vie : «My thoughts on me tyrannise ». Le terme anglais d' « imposthume » est intéressant à plusieurs titres. Formé à partir du verbe grec signifiant « séparer» (aphistanai), le vieux mot d'《 apostume » (latin : apostema) signifie au sens propre la séparation de matières corrompues formant un ulcère, soit un gonflement de matière purulente. C'est le nom ancien de l'abcès, du mot latin abcessus (ab-cedere) signifiant l'acte de se séparer ou de s'éloigner.

La notion centrale est donc celle de la séparation d'une partie (pars a toto) et de son pourrissement par sevrage. La tumeur enkystée dans la tête de Burton consiste bien, comme la pensée du roseau ou la pensée du membre pascaliens, en une partie séparée du tout - fragment psychisé du corps ou fragment corporéisé du psychisme - que son hypertrophie et sa suractivité morbide enflent aux dimensions d'un tout, semblable à un organisme enclavé dans un organisme dont il épuise les forces vives. Formation défensive contre un sevrage traumatique, repli et surinvestissement d'une infime partie d'un corps frappé de désintégration, l'«imposthume » de Burton, hypertrophié (tumidum) et infecté (foetum), retenant pour son propre usage et son propre accroissement (Steigerung) tout l'aliment libidinal, sèvre à son tour la personne des forces vitales de la libido : s'ensuivent un flux continu de cogitations morbides,

36. R. Burton, Anatomie de la Mélancolie, éd. Gisèle Vernet, Gallimard, « Folio classique », 2005 , p. 72.

37. Ferenczi, op. cit., p. 106. 
d'épouvantes sans raison, et un triste détachement de tous les plaisirs du monde (taedium vitae).

Mais la beauté saisissante de l'expression de Burton tient peut-être en premier lieu à sa graphie corrompue : «imposthume» pour apostume. On pense automatiquement à l'«un-heimlich» de Freud. L'im-posthume serait alors le mort nié comme mort, quelque chose de défunt non reconnu comme défunt, qui continuerait de vivre d'une vie fantomatique et dont la plainte perpétuelle aurait justement pour objet de faire « revenir » sans fin ce quelque chose de défunt pour exorciser le spectre d'une « cadavérisation du psychique ${ }^{38}$ ». Ce qui aurait dû devenir un bon objet interne (le bon objet maternel introjecté) s'est enkysté dans un organe que hante sa vie posthume. Dans la souffrance psychique de l'organe hypocondriaque - ici, la tête de Burton - pleure un moi mal incarné, monstrueux et méconnaissable, auquel son deuil impossible refuse de reposer en paix et, sous peine de trahir la mort psychique du tout, de se rendormir à jamais dans le silence de l'organisme. Dans le terme d' «imposthume» extrapolé par Burton, la vie psychique de l'organe est conçue littéralement comme mort-vivance du moi ou, pour le dire autrement, comme hantise pathogène et phénomène de revenance.

«Qu'on s'imagine un corps plein de membres pensants. » Ce corps composé de pensées qui en décompose le tout en une somme dispersée de cogitations morbides, doit-on vraiment l'imaginer? Ne l'a-t-on pas sous les yeux dès qu'on ouvre les Pensées, ce corpus inachevé, déchiré et décousu dont Emmanuel Martineau suggère qu'il dût être le résultat d'un découpage aux ciseaux opéré par Pascal sur les brouillons de plusieurs discours apologétiques ${ }^{39}$. L'œuvre est une somme de fragments, dont chacun est une pensée, illustrant le manque d'unité, le désordre, les disproportions et l'incomplétude nécessaire d'une science de l'« homme

38. « Dans le cas de l'hypocondrie, le psychique a pour fonction de conserver le mort (identification du mort à l'organe) en niant le mort, de le réanimer constamment et de garder ainsi sa puissance tutélaire (le psychique), d'éviter la cadavérisation du psychique équivalant à la décomposition » (Pierre Fédida, op. cit., p. 132-133).

39. Cf. la préface de l'éditeur à son édition des Pensées (Blaise Pascal, Discours sur la religion et sur quelques autres sujets, éd. Emmanuel Martineau, Armand Colin, 1992). 
sans Dieu ». L'anthropologie des Pensées, parce qu'elle entend décrire les hommes auxquels elle s'adresse, c'est-à-dire les esprits forts qui pensent se passer de Dieu, est une pathologie dont la formule est figurée dans l'éclatement du corpus : une hypocondrie généralisée dans laquelle chaque fragment - c'est-à-dire chaque pensée - est un organe affecté d'une cogitatio morbide pleurant l'unité perdue de la nature adamique. Bien plus qu'un livre inachevé, les Pensées sont inachevables, pour la raison évidente qu'une science de l'« homme sans Dieu » ne pourrait être totalisée sans trahir l'inachèvement de l'objet dont elle s'occupe. C'est une œuvre hypocondriaque, désintégrée en fragments ne pensant que des débris de vérités contradictoires, une œuvre hantée par le fantôme d'une totalité perdue que seule la théologie - une anthropologie de la grâce pourrait traduire dans un corpus aux parties articulées et aux membres réfléchissant le tout qui les unifie. En grand professionnel de l'art de persuader, Pascal sut renoncer à l'ouvrage apologétique qu'il avait entrepris d'écrire. Afin de mieux émouvoir le public d'incroyants auquel il s'adressait, il démembra son grand projet de Discours sur la religion chrétienne, le décapita de l'idée de Dieu et n'en donna à lire que les "membres pensants », somme désorganisée, corpus hypocondriaque tendant à l'« homme sans Dieu » le miroir en mille morceaux de ses méditations morbides.

Jean-Christophe Cavallin Professeur de littérature à l'université d'Aix-Marseille. 


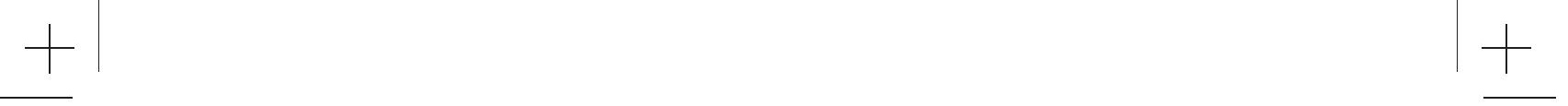

$+$ 